\title{
Alternative strategies for lumbar discectomy: intradiscal electrothermy and nucleoplasty
}

\author{
William C. Welch, M.D., and Peter C. Gerszten, M.D., M.P.H. \\ Departments of Neurological Surgery and Orthopaedic Surgery, and the School of Rehabilitative \\ Sciences, University of Pittsburgh Medical Center-Health System, Presbyterian University Hospital, \\ Pittsburgh, Pennsylvania
}

\begin{abstract}
In recent years the general trend in spinal surgery has been one of reductionism and minimalization. A number of techniques have recently been developed that are applicable in the treatment of lumbar disc herniation and discogenic pain due to degenerative disc disease. The purpose of this manuscript is to examine two newer percutaneous disc treatment techniques, intradiscal electrothermal therapy (IDET) anuloplasty and nucleoplasty. The authors review the appropriate clinical treatment criteria, techniques, and lessons learned after performing these procedures in more than 100 patients.

The IDET involves the percutaneous insertion of a specially designed thermal resistance probe followed by controlled heating of the intervertebral disc. This may result in disc shrinkage and reduction in pain. The nucleoplasty procedure involves the percutaneous removal of disc material by using a low-temperature resister probe to disintegrate and evacuate disc material, followed by thermal treatment of adjacent residual disc material. To date, no study has been published in which investigators examine the outcomes of this procedure for the treatment of radicular leg pain and low-back pain.

Both IDET and nucleoplasty appear to be safe procedures. The IDET procedure may be an alternative to lumbar interbody fusion. Although its long-term role is being defined, this technique appears to provide intermediate-term relief of pain in a population of patients with discogenic low-back pain. Nucleoplasty may provide a percutaneous alternative to microdiscectomy in selected cases.
\end{abstract}

\section{KEY WORDS • low-back pain • spinal fusion - intradiscal electrothermy $\bullet$ nucleoplasty}

The general trend in spinal surgery has been one of reductionism and minimalization, and has been driven by a number of factors, including surgeons' desires to reduce surgery-related trauma, patients' awareness of alternatives to open surgical procedures, and the development of new technologies. To this end, a number of techniques have been developed that are applicable to the treatment of lumbar disc herniation and discogenic pain due to degenerative disc disease.

These techniques have been designed either to shrink or remove disc material believed to be causing lumbar pain and/or radiculopathy. ${ }^{9}$ The most commonly examined and applied percutaneous techniques include arthroscopic microdiscectomy, automated percutaneous lumbar discectomy, and chymopapain, as well as other procedures. . $^{3,4,7,15,17}$ The purpose of this study is to examine two newer percutaneous disc treatment techniques, IDET anuloplasty and nucleoplasty. We review the appropriate clinical treatment criteria, techniques, and lessons learned after performing these procedures in more than 100 patients.

Abbreviations used in this paper: $\mathrm{ALIF}=$ anterior lumbar interbody fusion; IDET = intradiscal electrothermal therapy; SF-36 = Short Form-36; VAS = visual analog scale.

\section{REVIEW OF INTRADISCAL ELECTROTHERMAL THERAPY}

\section{Indications for Treatment}

The IDET procedure involves the percutaneous insertion of a specially designed thermal resistance probe, which is inserted through a cannula that is coiled into the selected area of targeted disc. After the location is confirmed using fluoroscopy, controlled heating of the disc material is performed $d^{5,10-12}$ which may result in disc-related shrinkage and reduction in pain (Fig. 1).,2,13,16 This procedure is supported by basic science research performed by investigators who assessed in vitro temperature mapping and demonstrated that thermal resistance catheter heating conducted temperatures sufficient to coagulate nerve endings and to contract collagen. ${ }^{1}$

A number of clinical studies have been performed, and are ongoing, to assess the efficacy of this device and procedure. These studies have included preclinical trials to examine the feasibility of the technique, as well as formal clinical trials ${ }^{2,5,10,11,12,14,17}$ (JA Saal, et al., unpublished data). We generally view IDET as an alternative to ALIF and apply the same diagnostic and treatment criteria. As with most treatments, patient selection may be the single most important criterion for a successful outcome. 


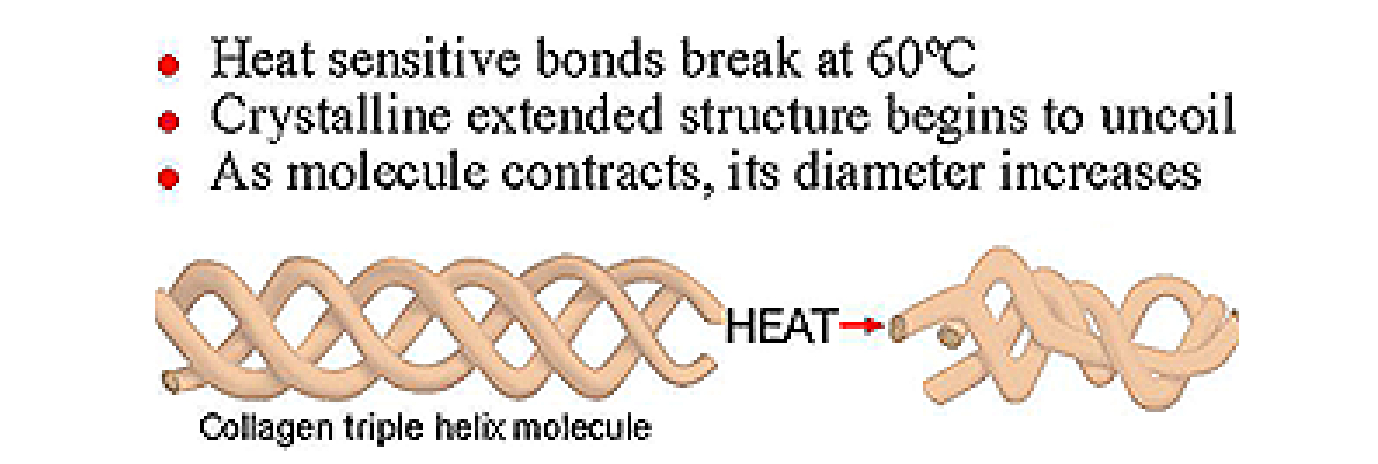

Fig. 1. Demonstration of the thermal modulation and shrinkage of collagen in response to IDET.

Discogenic low-back pain is the most common condition treated with IDET, and its presence has been our primary selection criterion. By discogenic pain, we refer to unremitting, persistent low-back pain that is worse with axial loading and improved with recumbency. Typically, the pain has been present for at least 6 months and is continuous in nature. The low-back pain is greater than leg pain. This is a nocioceptive pain and not a neuropathic pain syndrome.

Neuroimaging studies are necessary in the diagnostic evaluation process. Magnetic resonance imaging confirms the presence of disc degeneration, desiccation, high-intensity zones (Modic endplate changes), and loss of disc height. Contained disc fragments may also be present. Extruded and free fragments of disc cannot be effectively treated using this procedure, but patients with these lesions may be treated for discogenic low-back pain with the proviso that the fragments will be unchanged after treatment.

Provocative discography, conducted by an unbiased interventional neuroradiologist, is performed in most cases. This diagnostic study is used to confirm our clinical suspicions, and its results may be used as exclusionary criteria for patients with multilevel disease. Discography also provides the surgeon with technical information regarding placement of the intradiscal catheter. Specifically, if a skilled neuroradiologist has difficulty placing a 20 -gauge needle into the L5-S1 disc space, the surgeon can reasonably expect to have similar difficulties placing an even larger needle into the same intervertebral space.

Candidates for IDET will have undergone at least 6 months of nonsurgical treatments including physical therapy and nonsteroidal antiinflammatory drug therapy. Most patients will have undergone epidural steroid treatments and narcotic therapies prior to our evaluation. In summa-

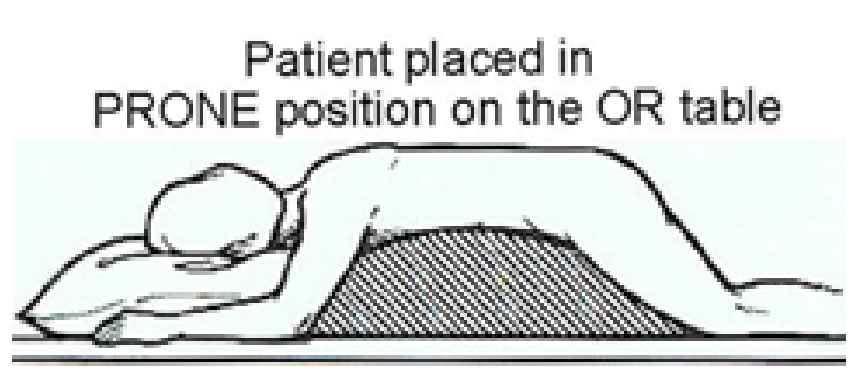

Fig. 2. Diagram illustrating patient positioning for IDET and nucleoplasty. ry, patients who generally meet all criteria for ALIF are offered IDET as a treatment alternative.

Another group of patients considered for IDET are those with multilevel degenerative disc disease in whom multilevel ALIF is being considered. These patients may benefit from combined-modality treatment including ALIF and IDET performed at selected levels. The use of IDET in this manner may avoid the need to fuse multiple levels of the lumbar spine sequentially.

Exclusion criteria include lumbar spinal instability that would require fusion, the presence of infection or malignancy at the level requiring treatment, severe psychosocial issues, or evidence of substance diversion. Multilevel disease (that is, three or more levels of disc disease documented on magnetic resonance imaging and discography) is a relative but not necessarily absolute contraindication to IDET anuloplasty. Greater than $75 \%$ loss of normal disc height is also a relative contraindication to the procedure.

\section{Treatment Technique}

We have used a standardized IDET technique in our last 75 patients. All procedures are performed in an operating room suite with the patient placed in a prone position on a Jackson table (Orthopaedic Systems, Inc., Union City, CA) or standard operating table with bolster support (Fig. 2 ). This allows the use of fluoroscopy in which there is lit-

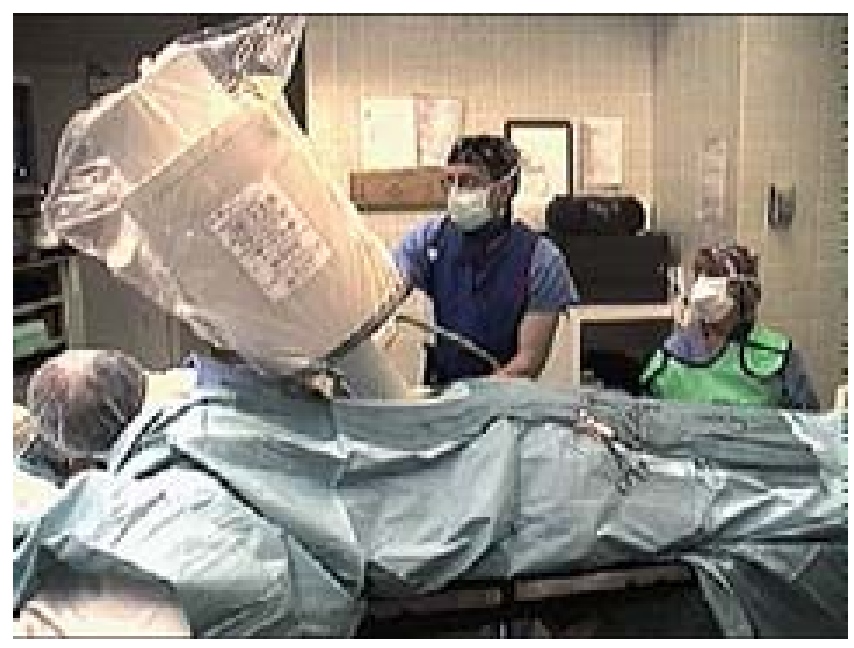

Fig. 3. Photograph demonstrating C-arm fluoroscopic use and operating room setup. 


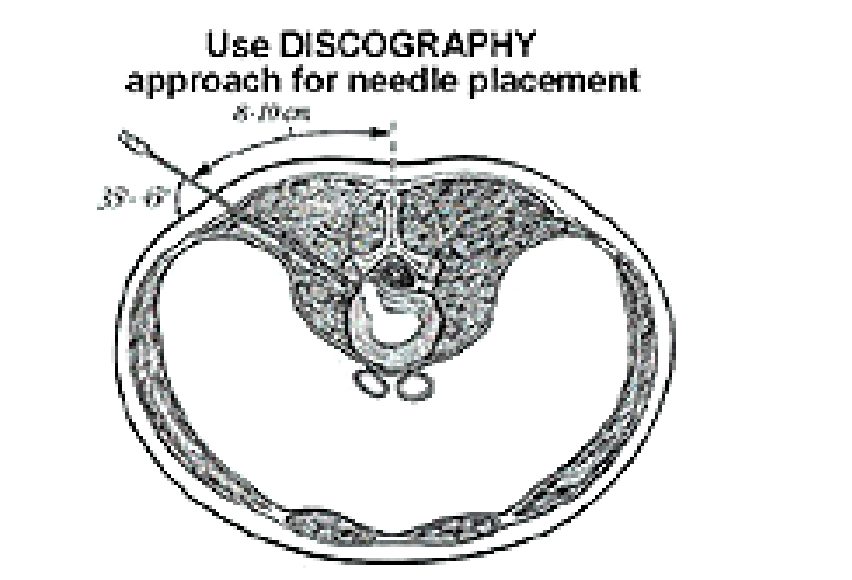

Fig. 4. Cross-section through lumbar area demonstrating entry point of the catheter.

tle radiographic obstruction. An anesthesiologist sedates the patient throughout the procedures. We use C-arm fluoroscopy to obtain anteroposterior, oblique ("needle eye view"), and lateral images (Fig. 3). The treatment level is localized, and a local anesthetic is applied to the skin 6 to $9 \mathrm{~cm}$ lateral to the midline on the side contralateral to the patient's pain. This approach provides excellent access to the disc space in most patients (Fig. 4). In this approach the anatomical triangular working zone described by Kambin, ${ }^{4}$ Parke, ${ }^{8}$ and O'Neill, et al.,${ }^{6}$ is used (Fig. 5). This "working triangle" avoids the exiting nerve root. Access to the L5-S1 intervertebral space may be difficult if high iliac crests or a very collapsed disc space are present. Difficulty at this level may be predicted based on the neuroradiologist's difficulty in accessing this space during preoperative discography.

The IDET set includes a 17-gauge needle and stylet (SpineCath; Smith \& Nephew Endoscopy [formerly
ORATEC Interventions, Inc.], Menlo Park, CA). These are directed toward the center of the disc under fluoroscopic guidance. The anulus is punctured, and the thermal resistance catheter is inserted through the needle into the disc (Fig. 6). The catheter is coiled within the disc under fluoroscopic guidance (Fig. 7). The tip of the catheter is directed toward the posterior aspect of the disc in such a manner that the heating elements of the catheter remain on the symptomatic side (Fig. 8). Once the catheter is successfully placed and its location is confirmed fluoroscopically, the catheter temperature is increased along an electronically programmed 13 -minute protocol to $90^{\circ} \mathrm{C}$ and allowed to remain at that temperature for 4 minutes. Antibiotic agents are not injected into the disc space. The catheter and needle are then removed as a single unit.

In patients with multilevel disease we follow the same protocol and treat each level with new catheters and needles. It has also been our routine to perform bilateral thermoplasty procedures in patients with only back pain at the diseased level. The patients are discharged after 2 hours of observation. Following the procedure, patients are instructed to resume, to the extent they can tolerate, their usual activities after 24 hours. We do not prescribe postoperative brace therapy, although this is recommended by other authors. ${ }^{12}$

\section{Treatment Difficulties and Complications}

Intradiscal electrothermal therapy appears to be a very safe procedure. Few complications have been reported, and our own experience has reflected this. The most common difficulty has been inserting the needle into the L5$\mathrm{S} 1$ disc space. In only rare cases can this level not be treated and the procedure aborted. In our experience there have been no postoperative infections or nerve root injuries.

Another treatment-related problem that may occur is difficulty in threading the catheter into the disc space once the needle is adequately positioned. Threading the cath-

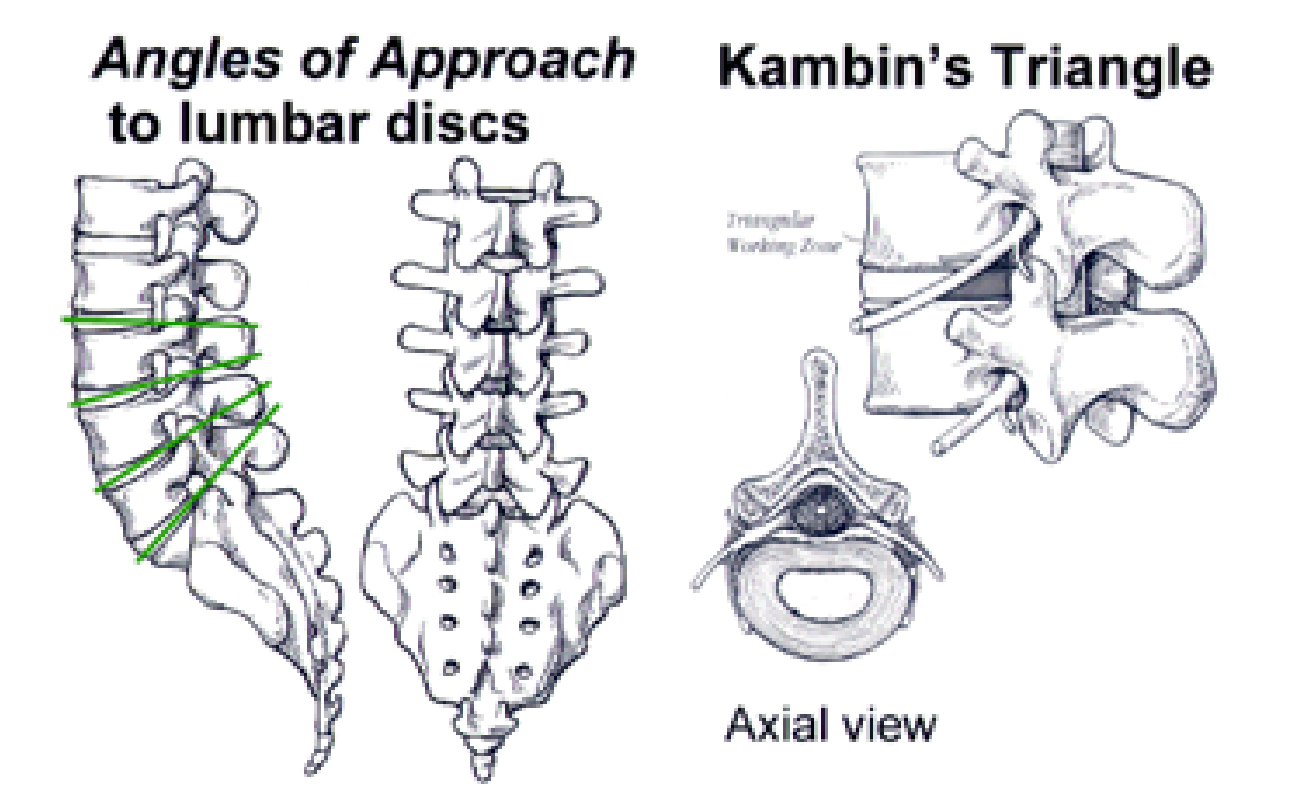

Fig. 5. Diagrams demonstrating the working triangles and safety zone for catheter placement. 


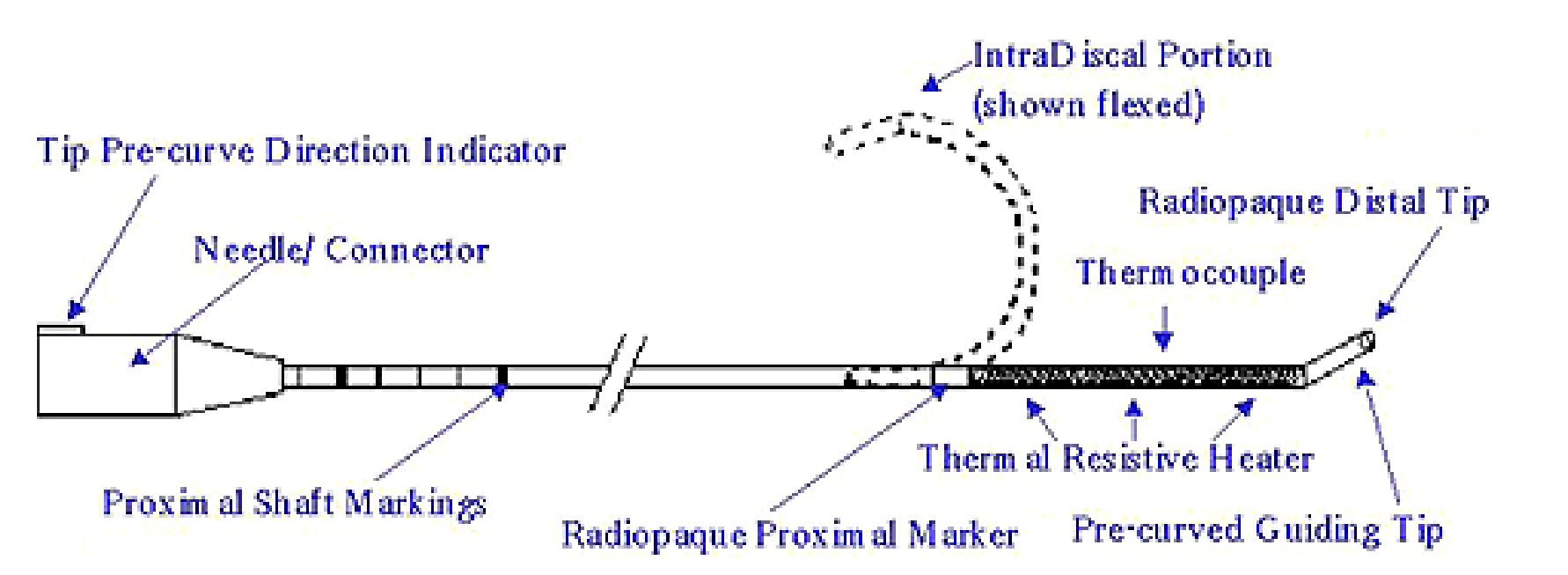

Fig. 6. Schematic drawing of IDET catheter.

eter may be difficult if the needle tip is positioned against the endplate or if the disc is extremely degenerated. Should this difficulty arise, it is extremely important not to force the catheter into the disc space because the catheter can be sheared off by the needle tip. Additionally, the catheter and needle must be removed as a single unit once the catheter is extended past the needle tip.

\section{Treatment-Related Results}

Presentations and publications in which authors have reviewed clinical outcomes of IDET-treated patients have

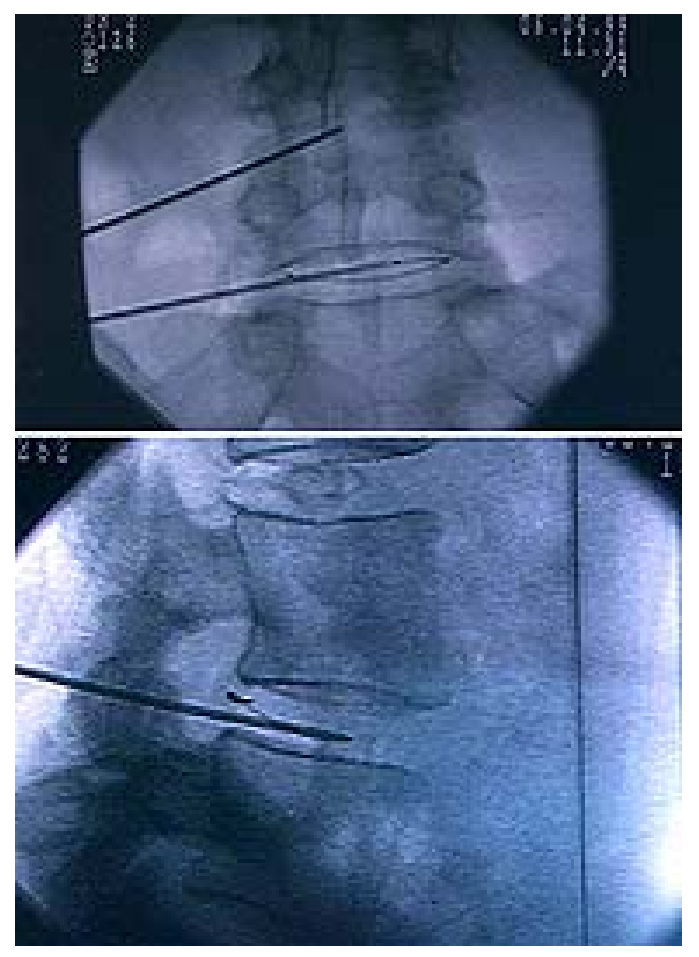

Fig. 7. Upper: Anteroposterior fluoroscopic image revealing the IDET catheter coiled in the disc space. Lower: Lateral fluoroscopic image demonstrating the IDET catheter coiled in the disc space. shown favorable results depending on their criteria. In most studies the investigators have used VAS scores, functional tolerance levels, and/or the SF-36 questionnaire. In most studies improvement in VAS scores, increase in functional tolerance levels, and improvements in SF-36 subscale scores have been demonstrated.

Generally positive outcomes in sitting scores and ability to work may be indicated posttreatment (Wetzel, et al., unpublished data). Some authors have demonstrated greater than $70 \%$ improvement in VAS pain scores and sitting tolerance in treated patients. ${ }^{2,12}$ Our favorable results (unpublished data) were similar to the those reported by others. ${ }^{2,11}$

In an initial 1-year follow-up evaluation of 27 patients who underwent IDET at our institution, the Oswestry Low Back Pain Disability Index scores improved in $75 \%$. The SF-36 Bodily Pain Subscale scores at 1 year improved in $52 \%$. We found no relationship between outcome and duration of symptoms, the number of levels treated, or receipt of Workers' compensation. No serious complications occurred in our series (unpublished data).

The authors of two unpublished studies have examined the potential effect of IDET on spinal stability. In a cadaveric study, Lee found no significant differences in nondestructive flexion-extension, lateral flexion, and axial rota-

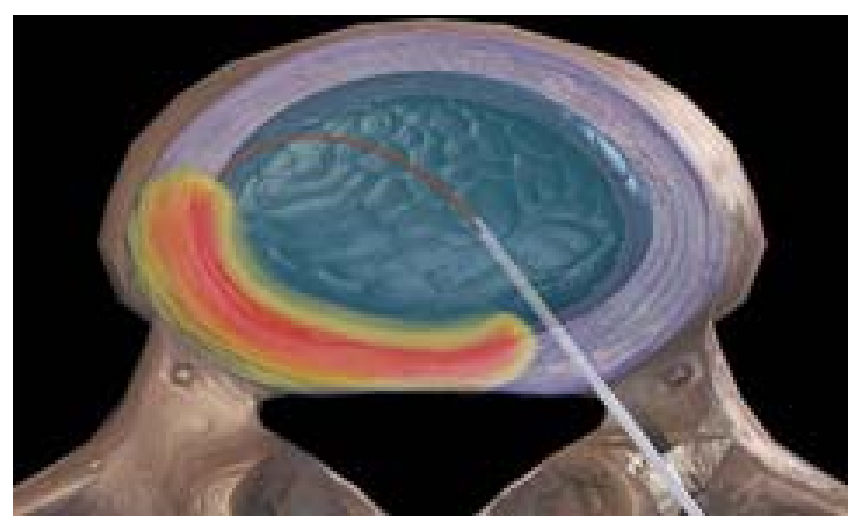

Fig. 8. Schematic drawing of the IDET cannula coiled in disc space, demonstrating heating zone. 


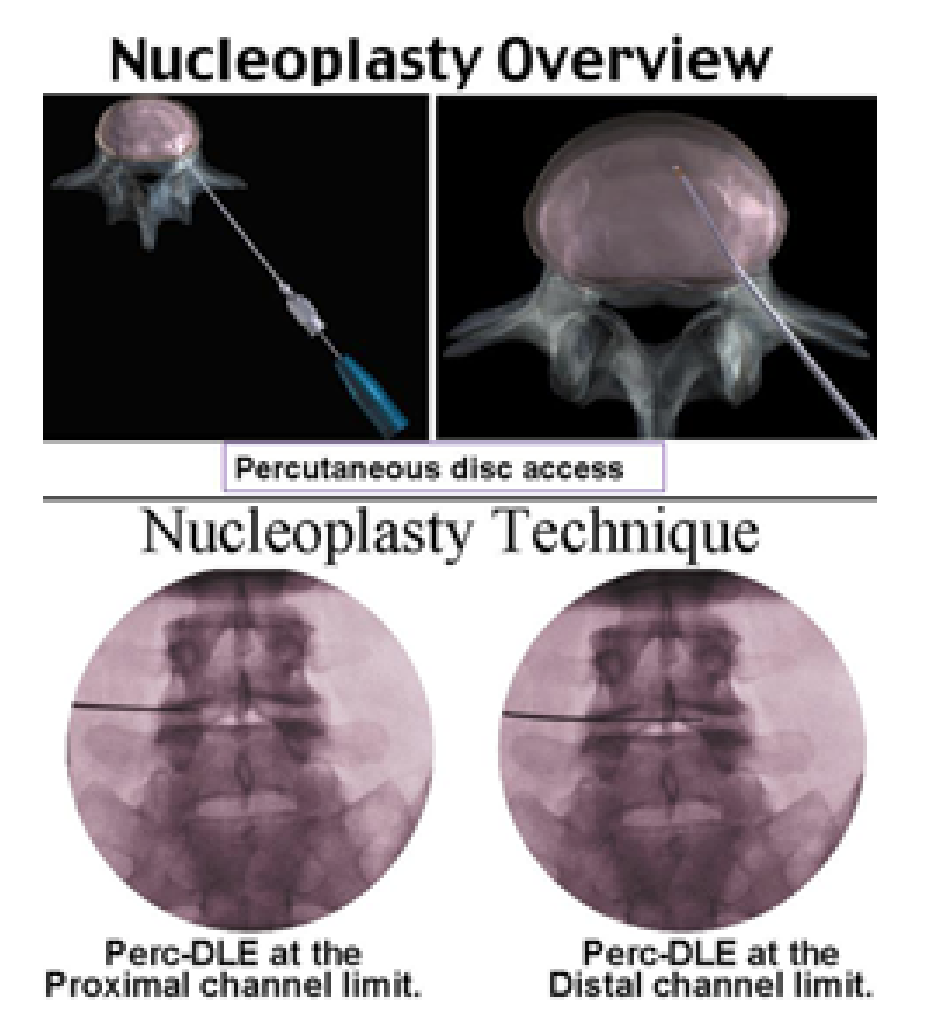

Fig. 9. Upper: Nucleoplasty catheter in the disc space. Lower: Anteroposterior fluoroscopic image demonstrating placement of the nucleoplasty catheter in the disc space.

tion biomechanics before and after treatment with IDET (unpublished data). In the other unpublished study, a retrospective clinical trial of 122 patients underwent IDET, the authors found that fewer than 5\% of IDET-treated patients might require open spinal surgery in the 6 to $18-$ month follow-up period (FP Laguttula, et al., unpublished data). These results suggest that IDET does not destabilize the spine.

\section{Summary of Intradiscal Electrothermal Therapy}

Intradiscal electrothermal therapy is a safe procedure

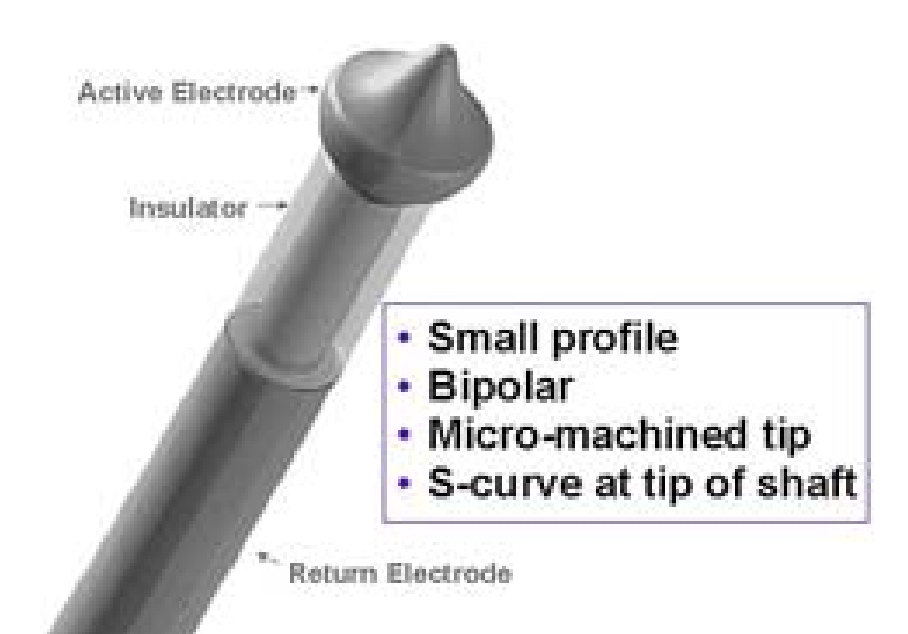

Fig. 10. Schematic drawing of the nucleoplasty catheter.

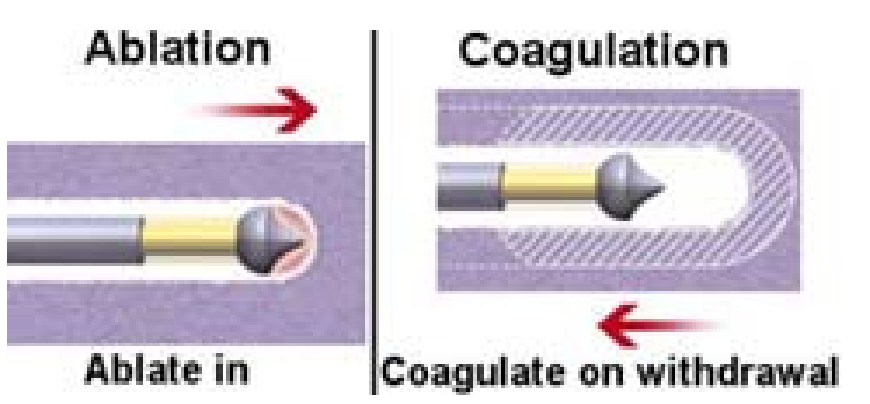

Fig. 11. Channeling during the nucleoplasty treatment.

that appears to be moderately effective in relieving pain of discogenic origin. It may be an alternative to lumbar interbody fusion in patients who cannot or wish not to undergo a major fusion procedure..$^{9,15,16,18}$ The complication rate is very low and recovery time is minimal, especially compared with an interbody fusion. Outcome in our personal series is comparable with most of the published results in that IDET was found to be effective in $75 \%$ of patients, resulting in improvement in low-back pain based on the Oswestry Disability Index as well as the SF-36 (unpublished data). Outcome does not appear to be dependent on the number of levels treated, duration of symptoms, or Workers' compensation. Finally, there is no evidence to suggest that IDET is harmful to patients, either clinically or biomechanically.

\section{REVIEW OF NUCLEOPLASTY}

Another percutaneous alternative procedure in the treatment of both discogenic low-back pain and radiculopathy due to contained disc ruptures is nucleoplasty (ArthroCare Corporation, Sunnyvale, CA). Nucleoplasty involves the percutaneous removal of disc material by using a lowtemperature resister probe to disintegrate and evacuate disc material, followed by thermal treatment of adjacent residual disc material. No research has been published to date in which authors have assessed the outcomes of this procedure for the treatment of radicular leg pain and lowback pain.

\section{Indications for Treatment}

The indications for nucleoplasty include discogenic

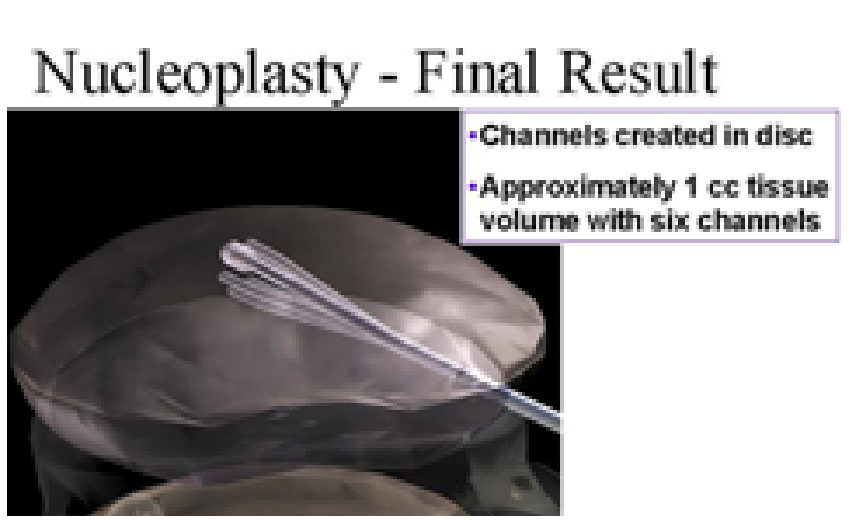

Fig. 12. Schematic drawing of channels created during nucleoplasty. 


\section{W. C. Welch and P. C. Gerszten}

low-back pain due to disc degeneration, as well as the treatment of lumbar disc bulges or disc ruptures causing radiculopathy. Contraindications include complete disc space collapse leading to inaccessibility of the intervertebral space, active disc space infection, and medical conditions that would preclude its safe performance.

\section{Treatment Technique}

Nucleoplasty involves the percutaneous insertion of a cannula positioned in the disc space in a manner similar to that for IDET (Fig. 9 upper). In contrast to IDET, the cannula is inserted on the same side as the patient's pain (that is, the side ipsilateral to the disc herniation). A catheter is then inserted via the needle into the disc space under fluoroscopic guidance and is positioned where the surgeon wants to ablate disc material (Fig. 9 lower).

The catheter has a low-temperature resister at its tip (Fig. 10). The resister generates a plasma field that disintegrates disc material into its hydrogen and oxygen constituents. The gases escape through the needle (Fig. 11). A localized area of increased temperature may result in the ablation of adjacent residual disc material. The tissue ablation and thermal treatment create a series of channels within the disc, reducing the pressure from the contained disc herniation or the nerve root and other pain generating structures (Fig. 12).

\section{Treatment-Related Results}

No published results were available for review. We have a personal experience with more than 25 patients who underwent nucleoplasty (unpublished results). Our preliminary experience is that nucleoplasty yields very positive results for relief of low-back pain and radiculopathy. Our best results have been in patients with diffuse disc protrusion and radicular pain, in whom very good relief of radicular pain occurs almost immediately after the procedure.

As with IDET, nucleoplasty appears to be safe. In our hands, there have been no infections, nerve root injuries, or worsening of symptoms after the procedure.

\section{Summary of Nucleoplasty}

Our preliminary experience is quite positive. Nucleoplasty seems to be a very good alternative to microdiscectomy in selected cases of contained disc herniations. Currently we are conducting a 1-year follow-up examination, using a collection of outcomes instruments, to obtain quality long-term outcome data.

\section{CONCLUSIONS}

Both IDET and nucleoplasty appear to be safe and effective procedures. The IDET procedure has gained some popularity and is being conducted in numerous centers. Its long-term role is being defined, but this procedure appears to provide successful intermediate-term pain relief in patients with discogenic low-back pain. Nucleoplasty may be a minimally invasive percutaneous alternative to microdiscectomy in selected cases.

\section{Disclaimer}

No grant, honorarium, consultation fee, fiduciary equivalent, or financial support was received for this work.

\section{References}

1. Ashley JE, Gharpuray VM, Saal JS, et al: Temperature distribution in the intervertebral disc: a comparison of intranuclear radio-frequency needle to a novel heating catheterl. BED 42: 77-78, 1999

2. Derby R, Eek B, Chen Y, et al: Intradiscal electrothermal annuloplasty (IDET): a novel approach for treating chronic discogenic back pain. Neuromodulation 3:82-88, 2000

3. Fritzell P, Hagg O, Wessberg P, et al: 2001 Volvo Award Winner in Clinical Studies: Lumbar fusion versus nonsurgical treatment for chronic low back pain: a multicenter randomized controlled trial from the Swedish Lumbar Spine Study Group. Spine 26:2521-2534, 2001

4. Kambin P: Alternative to open lumbar discectomy: arthroscopic microdiscectomy, in Welch WC, Jacobs GB, Jackson RP (eds): Operative Spine Surgery. Stamford, CT: Appleton \& Lange, 1999, pp 228-237

5. Karasek M, Bogduk N: Twelve-month follow-up of a controlled trial on intradiscal thermal anuloplasty for back pain due to internal disc disruption. Spine 25:2601-2607, 2000

6. O'Neill C, Derby R, Kenderes L: Precision injection techniques for diagnosis and treatment of lumbar disc disease. Semin Spine Surg 11:104-118, 1999

7. Onik G, Maroon J, Davis GW: Automated percutaneous discectomy at the L5-S1 level. Use of a curved cannula. Clin Orthop 238:71-76, 1989

8. Parke WW: Anatomy of the spinal nerve and its surrounding structures. Semin Orthop 6:65-71, 1991

9. Ray CD: Threaded fusion cages for lumbar interbody fusions: economic comparison with $360^{\circ}$ fusions. Spine 22:681-685, 1997

10. Saal JA, Saal JS: Intradiscal electrothermal treatment for chronic discogenic low back pain: a prospective outcome study with minimum 1-year follow-up. Spine 25:2622-2627, 2000

11. Saal JS, Saal JA: Intradiscal electrothermal therapy for the treatment of chronic discogenic low back pain. Oper Tech Orthop 10:271-281, 2000

12. Saal JS, Saal JA: Management of chronic discogenic low back pain with a thermal intradiscal catheter. A preliminary study. Spine 25:382-388, 2000

13. Schwarzer AC, Aprill CN, Derby R, et al: The relative contributions of the disc and zygapophyseal joint in chronic low back pain. Spine 19:801-806, 1994

14. Singh V: Intradiscal electrothermal therapy: a preliminary report. Pain Physic 3:367-373, 2000

15. Turner JA, Ersek M, Herron L, et al: Patient outcomes after lumbar spinal fusions. JAMA 268:907-911, 1992

16. Vamvanij V, Fredrickson BE, Thorpe JM, et al: Surgical treatment of internal disc disruption: an outcome study of four fusion techniques. J Spinal Disord 11:375-382, 1998

17. Welch WC, Gerszten PC, McGrath P: Intradiscal electrothermy: indications, techniques, and clinical results. Clin Neurosurg 48:219-225, 2001

18. Zdeblick TA: A prospective, randomized study of lumbar fusion. Preliminary results. Spine 18:983-991, 1993

Manuscript received June 15, 2002.

Accepted in final form July 19, 2002.

Address reprint requests to: William C. Welch, M.D., Department of Neurological Surgery, University of Pittsburgh Medical Center-Health System, Presbyterian University Hospital, Suite B400, 200 Lothrop Street, Pittsburgh, Pennsylvania 15213-2582. email:wwelch@neuronet.pitt.edu. 